\title{
Simulating spin waves in entropy stabilized oxides
}

\author{
Tom Berlijn $\odot,{ }^{1, *}$ Gonzalo Alvarez, ${ }^{2}$ David S. Parker, ${ }^{3}$ Raphaël P. Hermann, ${ }^{3}$ and Randy S. Fishman ${ }^{3}$ \\ ${ }^{1}$ Center for Nanophase Materials Sciences, Oak Ridge National Laboratory, Oak Ridge, Tennessee 37831, USA \\ ${ }^{2}$ Computational Sciences and Engineering Division, Oak Ridge National Laboratory, Oak Ridge, Tennessee 37831, USA \\ ${ }^{3}$ Materials Science and Technology Division, Oak Ridge National Laboratory, Oak Ridge, Tennessee 37831, USA
}

(Received 2 November 2020; revised 1 September 2021; accepted 3 September 2021; published 22 September 2021)

\begin{abstract}
The entropy stabilized oxide $\mathrm{Mg}_{0.2} \mathrm{Co}_{0.2} \mathrm{Ni}_{0.2} \mathrm{Cu}_{0.2} \mathrm{Zn}_{0.2} \mathrm{O}$ exhibits antiferromagnetic order and magnetic excitations, as revealed by recent neutron scattering experiments. This observation raises the question of the nature of spin-wave excitations in such disordered systems. Here, we investigate theoretically the magnetic ground state and the spin-wave excitations using linear spin-wave theory in combination with the supercell approximation to take into account the extreme disorder in this magnetic system. We find that the experimentally observed antiferromagnetic structure can be stabilized by a rhombohedral distortion together with large secondnearest-neighbor interactions. Our calculations show that the spin-wave spectrum consists of a well-defined low energy coherent spectrum in the background of an incoherent continuum that extends to higher energies.
\end{abstract}

DOI: 10.1103/PhysRevResearch.3.033273

\section{INTRODUCTION}

Entropy stabilization means that a single phase is stabilized by the mixing entropy gained from randomly distributing a number of elements, typically five or more, over a single crystal lattice [1]. In 2015 a new family was added to the class of high entropy materials with the discovery of the high entropy oxides (HEOs). By mixing equimolar amounts of $\mathrm{MgO}, \mathrm{CoO}, \mathrm{NiO}, \mathrm{CuO}$, and $\mathrm{ZnO}$, Rost et al. [2] found that $\mathrm{Mg}_{0.2} \mathrm{Co}_{0.2} \mathrm{Ni}_{0.2} \mathrm{Cu}_{0.2} \mathrm{Zn}_{0.2} \mathrm{O}$ (hereafter $\mathrm{MgO}-\mathrm{HEO}$ ) could be stabilized in a simple rock-salt structure in which the oxygen atoms occupy one of the face-centered cubic (fcc) sublattices and the cations are randomly distributed over the other fcc sublattice. The entropy stabilized nature of the phase is apparent when considering that $\mathrm{CuO}$ and $\mathrm{ZnO}$ do not form in the rock-salt structure that forces $\mathrm{Cu}$ and $\mathrm{Zn}$ into octahedral coordination. Rost et al. demonstrated that the sample could be switched between a multiphase structure and the aforementioned single phase rock-salt structure by repeated heating and cooling, thereby proving the reversibility of the entropy-driven transition. Furthermore, a combination of $\mathrm{x}$ ray diffraction, $\mathrm{x}$-ray absorption fine structure, and scanning transmission electron microscopy with energy dispersive $\mathrm{x}$ ray spectroscopy showed that the $\mathrm{MgO}-\mathrm{HEO}$ sample was chemically and structurally homogeneous [2]. The HEOs are not only interesting from a basic scientific point of view, but also exhibit promising functional properties, such as high Li-ion storage capacity and cycling stability [3], colossal di-

\footnotetext{
*berlijnt@ornl.gov

Published by the American Physical Society under the terms of the Creative Commons Attribution 4.0 International license. Further distribution of this work must maintain attribution to the author(s) and the published article's title, journal citation, and DOI.
}

electric constants [4], superionic conductivity [5], and a high ratio of elastic modulus to thermal conductivity [6]. In addition to rock-salt HEOs, HEOs with perovskite [7-9], fluorite [10,11], and spinel [12] structures have been discovered, together with high entropy carbides [13,14], borides [15], and chalcogenides [16].

The magnetic properties of $\mathrm{MgO}-\mathrm{HEO}$ had remained largely unexplored, until recently. Exchange bias measurements of permalloy/MgO-HEO heterostructures revealed that $\mathrm{MgO}-\mathrm{HEO}$ is an antiferromagnet [17]. In two subsequent studies, neutron diffraction and magnetic susceptibility measurements have shown that bulk MgO-HEO displays antiferromagnetic (AFM) order with Néel temperatures reported to be 113 [18] and $120 \mathrm{~K}$ [19], with magnetic moments arranged in the same AFM ground state as $\mathrm{NiO}$ and $\mathrm{CoO}$ $[20,21]$. In this so-called AFM order of the second kind, spins are parallel within the (111) planes and antiparallel between the (111) planes (see Fig. 1). Inelastic neutron scattering measurements have shown that spin-wave excitations are gapped, with a spin gap on the order of $\approx 7 \mathrm{meV}$ [22]. The gap is gradually buried in quasielastic fluctuations upon heating towards $T_{N}$. Interestingly, the spin-wave excitations persist above $T_{N}$, up to room temperature [18]. Unlike in binary oxides such as $\mathrm{NiO}$ and $\mathrm{CoO}$, no strong lambda anomaly has been observed in the specific heat $[18,19]$. Recent muon spin relaxation measurements [22] indicate that $\mathrm{MgO}-\mathrm{HEO}$ undergoes a broad continuous AFM phase transition starting at $140 \mathrm{~K}$ and being fully ordered at $100 \mathrm{~K}$, explaining why the specific heat anomaly is very weak and broad. The discovery of magnetic long-range order in MgO-HEO leads to a number of questions. How can the experimentally observed AFM long-range order be stabilized in the presence of the randomly distributed moments of $\mathrm{Co}, \mathrm{Ni}$, and $\mathrm{Cu}$ and the $40 \%$ of spin vacancies created by the nonmagnetic $\mathrm{Mg}$ and $\mathrm{Zn}$ ions? What is the nature of spin-wave excitations in such an extremely disordered magnetic environment? How 


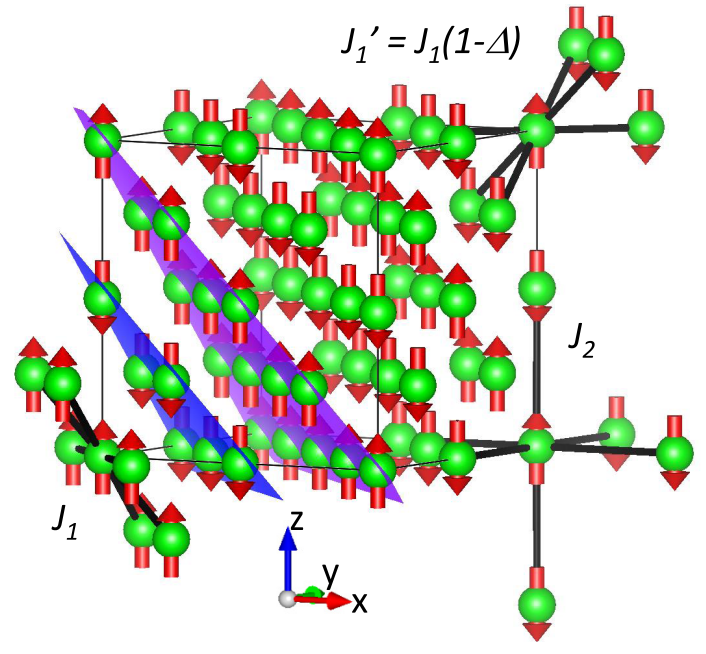

FIG. 1. Spins on a face centered cubic lattice arranged in the antiferromagnetic order of the second kind with ordering wave vector $q=(1 / 2,1 / 2,1 / 2)$ in which the spins are within/between the (111) planes and aligned ferromagnetically/antiferromagnetically. Under the rhombohedral distortion, the nearest-neighbor exchange coupling is split into $J_{1} / J_{1}^{\prime}$ between spins within/between the (111) planes, whereas the second-nearest-neighbor exchange coupling $J_{2}$ is not split.

do the magnetic properties of $\mathrm{MgO}-\mathrm{HEO}$ compare with those of $\mathrm{Ni}_{1-x} \mathrm{Mg}_{x} \mathrm{O}$ [23], $\mathrm{Ni}_{1-x} \mathrm{Zn}_{x} \mathrm{O}$ [24], and $\mathrm{Co}_{1-x} \mathrm{Mg}_{x} \mathrm{O}$ [25], which also display antiferromagnetic order in the presence of similarly large spin-vacancy concentrations?

In this paper, we theoretically investigate the magnetic ground state and spin-wave excitations in the high entropy oxide $\mathrm{MgO}$-HEO. We model the $\mathrm{Co}^{2+}, \mathrm{Ni}^{2+}$, and $\mathrm{Cu}^{2+}$ cations with spins of size $3 / 2,1$, and $1 / 2$, respectively, and the $\mathrm{Mg}^{2+}$ and $\mathrm{Zn}^{2+}$ cations as being spin vacancies. We randomly distribute the cations on a rhombohedrally distorted facecentered cubic lattice and analyze the magnetic properties via linear spin-wave theory in combination with the supercell approximation. We find that large next-nearest-neighbor exchange couplings and large rhombohedral distortions are required in order to stabilize the experimentally observed antiferromagnetic ground state with propagation vector $q=$ $(1 / 2,1 / 2,1 / 2)$. The spin-wave excitation spectrum of our model for $\mathrm{MgO}-\mathrm{HEO}$ consists of a coherent component at low energies and an incoherent component at high energies. We also model $\mathrm{Co}_{0.33} \mathrm{Ni}_{0.33} \mathrm{Cu}_{0.33} \mathrm{O}$ containing moment-size disorder only and $\mathrm{Ni}_{0.6} \mathrm{Mg}_{0.4} \mathrm{O}$ containing spin-vacancy disorder only. We find qualitative differences in the spin-wave spectra of these systems compared with that of MgO-HEO. Finally we also investigate the influence of disorder on the spin-wave gap and find that it is proportional to the average moment size per lattice site.

\section{METHODS}

In order to qualitatively investigate the ground state and spin-wave excitations in $\mathrm{MgO}-\mathrm{HEO}$, we use a simplified model. X-ray absorption spectroscopy [18] and extended Xray absorption fine structure [26] are indicative of the cations in $\mathrm{MgO}-\mathrm{HEO}$ being divalent. In addition, electron paramag- netic resonance [19] and density functional theory (DFT) [27] have shown that $\mathrm{Co}^{2+}$ is in the high spin state. Based on these experimental and theoretical results, we treat the $\mathrm{Zn}^{2+}$ and $\mathrm{Mg}^{2+}$ cations as spin vacancies and the $\mathrm{Co}^{2+}, \mathrm{Ni}^{2+}$ and $\mathrm{Cu}^{2+}$ cations as spins with moment sizes $3 / 2,1$, and $1 / 2$, respectively, ignoring orbital contributions here. To describe the interactions between the magnetic moments, we take into account the nearest- and next-nearest-neighbor exchange couplings. In the rock-salt structure, the nearest-neighbor cations are connected through a cation-oxygen-cation group that has a bond angle of $90^{\circ}$. For the second-neighboring cations, the mediating oxygen ions are located in between the two cations, such that the cation-oxygen-cation bond angle is $180^{\circ}$. Therefore, according to the Goodenough-Kanamori rules for superexchange [28,29], the nearest-neighbor spin interactions are ferromagnetic and the next-nearest-neighbor interactions are stronger and antiferromagnetic. This is also what has been concluded from DFT calculations for $\mathrm{CoO}$ [30], $\mathrm{NiO}$ [31], and MgO-HEO [32] and from some inelastic neutron scattering studies for $\mathrm{NiO}$ [33] and $\mathrm{CoO}$ [34]. Finally, we incorporate a rhombohedral distortion into our model. On an undistorted fcc lattice the antiferromagnetic order of the second kind will be a mixture of four degenerate ordering wave vectors: $q=(1 / 2,1 / 2,1 / 2),(-1 / 2,1 / 2,1 / 2)$, $(1 / 2,-1 / 2,1 / 2)$, and $(1 / 2,1 / 2,-1 / 2)$. A rhombohedral distortion with the trigonal axis along the [111] direction will naturally stabilize the $q=(1 / 2,1 / 2,1 / 2)$ configuration in favor of the other three ordering wave vectors [35]. Putting it all together, we consider the following model:

$$
\begin{aligned}
H= & -J_{1} \sum_{\left\langle\left\langle r, r^{\prime}\right\rangle\right\rangle_{1 p}} \hat{\mathbf{S}}_{r} \cdot \hat{\mathbf{S}}_{r^{\prime}}-J_{1}^{\prime} \sum_{\left\langle\left\langle r, r^{\prime}\right\rangle\right\rangle_{1 a}} \hat{\mathbf{S}}_{r} \cdot \hat{\mathbf{S}}_{r^{\prime}} \\
& -J_{2} \sum_{\left\langle\left\langle r, r^{\prime}\right\rangle\right\rangle_{2}} \hat{\mathbf{S}}_{r} \cdot \hat{\mathbf{S}}_{r^{\prime}}-K \sum_{r} S_{r z}^{2}
\end{aligned}
$$

where $r$ labels the sites on the disordered cation fcc lattice, $\left\langle\left\langle r, r^{\prime}\right\rangle\right\rangle_{1 p}$ labels the first-nearest neighbors within the ferromagnetic (111) planes, $\left\langle\left\langle r, r^{\prime}\right\rangle\right\rangle_{1 a}$ labels the first-nearest neighbors between the ferromagnetic (111) planes, and $\left\langle\left\langle r, r^{\prime}\right\rangle\right\rangle_{2}$ labels the second-nearest neighbors. Overall our simplified model depends on four parameters, $J_{1}^{\prime}=J_{1}(1-\Delta)$, $J_{2}$, and $K$, where $\Delta$ quantifies the strength of the rhombohedral distortion and $K$ quantifies the magnetic anisotropy. This model captures the disorder due to the random distributions of the $\mathrm{Co}^{2+}, \mathrm{Ni}^{2+}$, and $\mathrm{Cu}^{2+}$ moment sizes and treats the $\mathrm{Mg}^{2+}$ and $\mathrm{Zn}^{2+}$ equivalently as spin vacancies. For simplicity, disorder in the magnetic exchange couplings [32] has been ignored.

To study the spin-wave excitation spectra of our model for $\mathrm{MgO}-\mathrm{HEO}$, we use a combination of linear spin-wave theory [36] and the supercell approximation [37]. The linearspin-wave formalism consists of two steps. First the classical ground state configuration is determined. To this end we use the conjugate gradient method as implemented in the SpinGlassSW computer program [38]. In the second step, the classical ground state is used to perform a Holstein-Primakoff transformation so that our interacting quantum-spin model (1) can be approximated as a quadratic boson Hamiltonian that can be solved with exact diagonalization. To treat the strong magnetic disorder in our model for $\mathrm{MgO}-\mathrm{HEO}$, we 


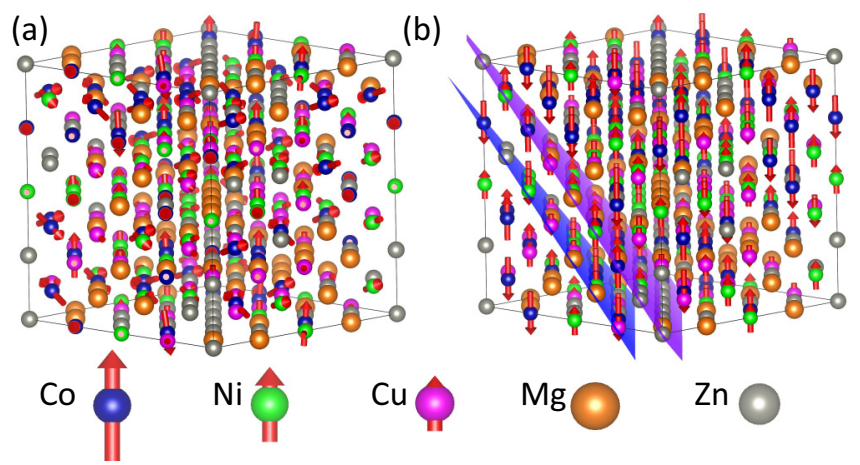

FIG. 2. Magnetic ground states for a $\mathrm{Co}_{52} \mathrm{Ni}_{52} \mathrm{Cu}_{52} \mathrm{Mg}_{52} \mathrm{Zn}_{48}$ supercell in the case of isotropic interactions $(K=0)$ with (a) $J_{2}=$ $-J_{1}, \Delta=0$ and (b) $J_{2}=-15 J_{1}, \Delta=0.5$.

use the supercell approximation in which the disordered system is approximated by a large number of supercells, within which the cations are disordered but beyond which they are artificially periodic. For other studies using linear spin-wave theory in combination with the supercell approximation, we refer to Refs. [39,40], and for alternative methods to simulate magnetic excitations in disordered systems we refer to Refs. [41,42].

\section{GROUND STATE}

Before studying the spin-wave excitations in MgO-HEO, we first focus on the stability of the $q=(1 / 2,1 / 2,1 / 2)$ AFM ground state depicted in Fig. 1. In the ordered fcc spin lattice this AFM ground state is stabilized in the parameter regime $\left|J_{2} / J_{1}\right|>1-\Delta$ [35]. Here $J_{1}>0$ is the FM nearest- and $J_{2}<0$ is the AFM next-nearest-neighbor exchange coupling, and $\Delta$ parametrizes the strength of the rhombohedral distortion (see Fig. 1). From this inequality we can see that the $q=(1 / 2,1 / 2,1 / 2)$ AFM ground state becomes more stable upon increasing the magnitude of the second-nearest exchange coupling and the rhombohedral distortion. Figure 1 shows that this makes intuitive sense. By inducing a rhombohedral distortion, the magnitude of the FM exchanges within the (111) planes $\left(J_{1}\right)$ will increase relative to those between the (111) planes $\left[J_{1}^{\prime}=J_{1}(1-\Delta)\right]$. The AFM $J_{2}$ exchange only couples moments between the (111) planes and therefore helps to further stabilize the $q=(1 / 2,1 / 2,1 / 2)$ AFM ground state.

In the presence of disorder, the stability arguments for the $q=(1 / 2,1 / 2,1 / 2)$ AFM ground state qualitatively work the same. In Fig. 2 we consider a $4 \times 4 \times 4$ orthogonal supercell relative to the conventional fcc unit cell. This supercell contains $52 S=3 / 2,52 S=1$, and $52 S=1 / 2$ moments corresponding to the randomly positioned $\mathrm{Co}, \mathrm{Ni}$, and $\mathrm{Cu}$ cations, respectively. That leaves 100 randomly distributed spin vacancies corresponding to the $\mathrm{Mg}$ and $\mathrm{Zn}$ cation sites. Figure 2(a) illustrates that for $J_{2}=J_{1}$ and $\Delta=0$, the ground state is in some noncollinear configuration. Upon increasing the second-nearest exchange to $J_{2}=-15 J_{1}$ and the rhombohedral distortion to $\Delta=0.5$, the $q=(1 / 2,1 / 2,1 / 2)$ AFM configuration is stabilized despite the disorder [see Fig. 2(b)]. We note that the positions of the moments within the super-
TABLE I. Percentage of orthogonal $\mathrm{Co}_{52} \mathrm{Ni}_{52} \mathrm{Cu}_{52} \mathrm{Mg}_{52} \mathrm{Zn}_{48}$ supercells being in the $q=(1 / 2,1 / 2,1 / 2)$ AFM ground state in the case of isotropic interactions: $K=0$, obtained from simulating 100 $\mathrm{Co}_{52} \mathrm{Ni}_{52} \mathrm{Cu}_{52} \mathrm{Mg}_{52} \mathrm{Zn}_{48}$ supercells.

\begin{tabular}{ccccc}
\hline \hline & $\begin{array}{c}J_{2}=-J_{1} \\
(\%)\end{array}$ & $\begin{array}{c}J_{2}=-2 J_{1} \\
(\%)\end{array}$ & $\begin{array}{c}J_{2}=-5 J_{1} \\
(\%)\end{array}$ & $\begin{array}{c}J_{2}=-15 J_{1} \\
(\%)\end{array}$ \\
\hline$\Delta=0.0$ & 0 & 0 & 0 & 6 \\
$\Delta=0.1$ & 0 & 0 & 0 & 42 \\
$\Delta=0.2$ & 0 & 0 & 20 & 81 \\
$\Delta=0.5$ & 2 & 65 & 94 & 95 \\
\hline \hline
\end{tabular}

cells in this paper have been randomized under the constraint that in the $q=(1 / 2,1 / 2,1 / 2)$ AFM configuration, the total moment is zero. To accomplish this, we divide the fcc lattice into two sets of parallel (111) planes. We distribute the cations in such a way that the sum of the total moments in the planes for which in the $q=(1 / 2,1 / 2,1 / 2)$ AFM state the moments are up [e.g., the purple plane in Fig. 2(b)] is equal to the sum of the total moments in the planes for which in the $q=(1 / 2,1 / 2,1 / 2)$ AFM state the moments are down [e.g., the blue plane in Fig. 2(b)]. We note that in this paper we have chosen the number of cations of each species to be even. In principle that constraint could have been relaxed to reach distributions closer to the equiatomic limit.

We now quantitatively investigate the stability of the $q=$ $(1 / 2,1 / 2,1 / 2)$ AFM ground state by varying the secondneighbor exchange coupling and the rhombohedral distortion parameter over a range of values. For each of the $4 \times 4$ parameter combinations listed in Table I, we simulate 100 orthogonal disordered $\mathrm{Co}_{52} \mathrm{Ni}_{52} \mathrm{Cu}_{52} \mathrm{Mg}_{52} \mathrm{Zn}_{48}$ supercells, again under the constraint that the total moment is zero in the $q=(1 / 2,1 / 2,1 / 2)$ AFM configuration. For each supercell, we minimize the classical energy via the conjugate gradient method, starting from 20 different random spin configurations. The configuration with the lowest energy is assigned to be the ground state. The average percentage of energy minimizations for which the ground state is found strongly depends on the model parameters. Reaching the ground state is the hardest for $\Delta=0.0$ and $J_{2}=-15 J_{1}$ for which the lowest energy is typically reached only in two of the 20 energy minimizations. For $\Delta=0.5$ and $J_{2}=-15 J_{1}$ on the other hand the ground state configuration is typically found in eight of the 20 energy minimizations. In total $4 \times 4 \times 100 \times 20=$ 32000 energy minimizations have been performed to produce Table I. To determine whether the ground state energy corresponds to the $q=(1 / 2,1 / 2,1 / 2)$ AFM configuration, we considered the average of the absolute difference for each of the spin vectors in these configurations. Appendix A contains more details about how we differentiate spin configurations.

As expected, the percentage of cases for which the $q=$ $(1 / 2,1 / 2,1 / 2)$ AFM ground state is realized increases as a function of the next-nearest exchange coupling $J_{2}$ and the rhombohedral distortion $\Delta$ (see Table I). Yet the strength of the parameters required to stabilize the $q=(1 / 2,1 / 2,1 / 2)$ AFM ground state is considerably higher than for the ordered case, due to the disorder. For example, for the ordered case with $\Delta=0.1, J_{2}=-0.9 J_{1}$ is sufficient. Our model for the 


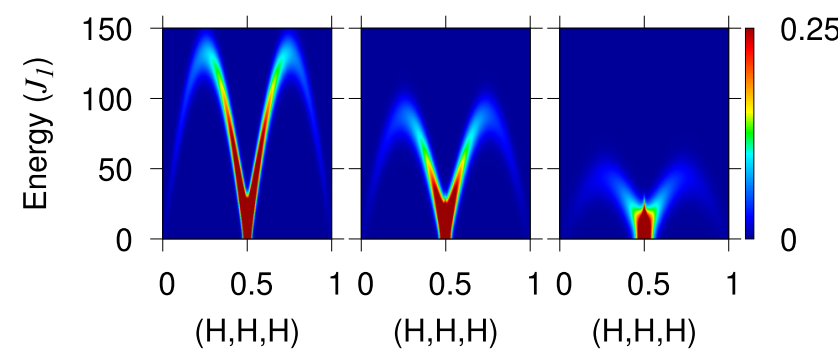

FIG. 3. Spin-wave spectra of ordered $\mathrm{CoO}$ (left), $\mathrm{NiO}$ (middle), and $\mathrm{CuO}$ (right) fcc lattice spin models with $J_{2}=-15 J_{1}, \Delta=0.5$, and $K=0$.

disordered MgO-HEO with $\Delta=0.1$ and $J_{2}=-15 J_{1}$ realizes the $q=(1 / 2,1 / 2,1 / 2)$ AFM ground state in only $42 \%$ of the configurations. Another interesting observation is that for $\Delta=0$ and $J_{2}=-15 J_{1}, 6 \%$ of the configurations resides in the $q=(1 / 2,1 / 2,1 / 2)$ AFM ground state, even without a rhombohedral distortion. To investigate the influence of anisotropy, we have performed the same energy minimization procedure for a set of smaller $\mathrm{Co}_{6} \mathrm{Ni}_{6} \mathrm{Cu}_{6} \mathrm{Mg}_{6} \mathrm{Zn}_{8}$ supercells. From comparing Tables III and IV, we see that the number of configurations, for which the $q=(1 / 2,1 / 2,1 / 2)$ AFM ground state is realized, is essentially the same for the isotropic case $(K=0)$ and the anisotropic case $\left(K=0.05 J_{1}\right)$, respectively.

\section{SPIN-WAVE EXCITATIONS}

Having understood the stability regime of the classical $q=(1 / 2,1 / 2,1 / 2)$ AFM ground state for our MgO-HEO model, we move on to study the corresponding spin-wave excitation spectrum. To that end we will focus on the parameters $J_{2}=-15 J_{1}$ and $\Delta=0.5$ and assume the ground state to be the $q=(1 / 2,1 / 2,1 / 2)$ AFM configuration. Before analyzing the influence of disorder in our MgO-HEO model, it is instructive to consider the spin-wave spectra of the ordered $\mathrm{CoO}, \mathrm{NiO}$, and $\mathrm{CuO}$ fcc spin models shown in Fig. 3. We notice two things. First, the energy range of the spin-wave spectra is proportional to the moment magnitude, $S=3 / 2,1$, and $1 / 2$, of the $\mathrm{Co}, \mathrm{Ni}$, and $\mathrm{Cu}$ cations, respectively. Second, we convoluted the spin-wave spectra with a Gaussian of width $0.5 J_{1}$ for the purpose of visualization. Other than that, the spin-wave bands in Fig. 3 are sharp in momentum and energy space.

In Fig. 4 we present the spin-wave spectra for our MgOHEO model. These spectra are obtained from averaging over 100 configurations, each containing $250 \mathrm{fcc}$ lattice sites on average, over which the $\mathrm{Co}, \mathrm{Ni}$, and $\mathrm{Cu}$ moments and the $\mathrm{Mg}$ and $\mathrm{Zn}$ spin vacancies are randomly distributed. We again use the constraint described in the previous section that the total moment vanishes in the $q=(1 / 2,1 / 2,1 / 2)$ AFM state. To better average the disorder configurations, we not only randomly distribute the cations within the supercells, but also randomly change the shapes of the supercells themselves, as Appendix B explains. Comparing the spin-wave spectrum of the strongly disordered MgO-HEO model in Fig. 4 to those of our models for the ordered $\mathrm{CoO}, \mathrm{NiO}$, and $\mathrm{CuO}$ systems in Fig. 3 reveals notable differences. First, the spin-wave

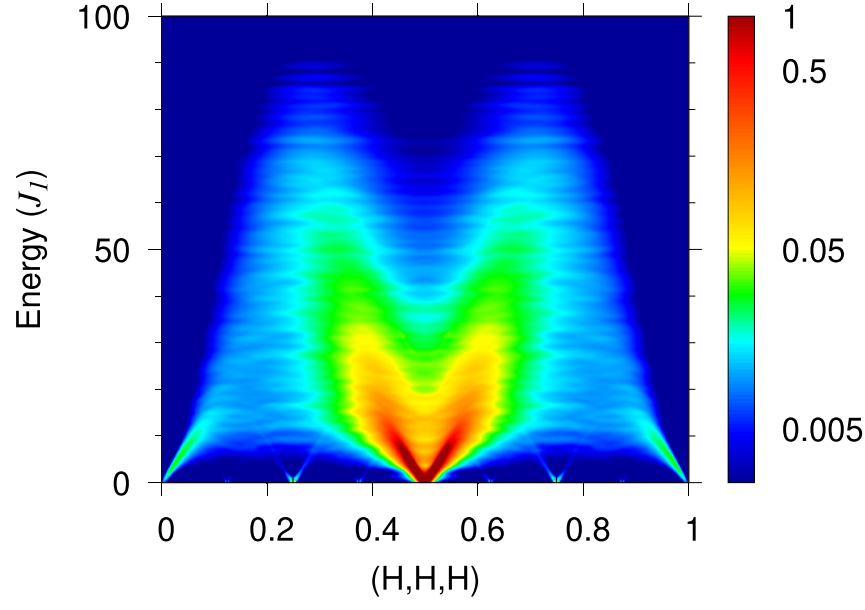

FIG. 4. Spin-wave spectrum of the $\mathrm{Mg}_{0.2} \mathrm{Co}_{0.2} \mathrm{Ni}_{0.2} \mathrm{Cu}_{0.2} \mathrm{Zn}_{0.2} \mathrm{O}$ fcc lattice spin model with moment size and spin vacancy disorder, obtained from averaging over 100 nonorthogonal supercells with 250 fcc lattice sites on average and $J_{2}=-15 J_{1}, \Delta=0.5$, and $K=0$.

spectrum of the MgO-HEO model displays large broadenings in energy and momentum space corresponding to the short lifetimes and short mean free paths of the spin waves due to the presence of strong disorder.

Another striking difference is that for the MgO-HEO model the spin-wave spectrum can be characterized by two regions. In the low energy regime, roughly within $[0,10] J_{1}$, the spin-wave spectrum exhibits a relatively sharp dispersion. At higher energies, roughly within $[10,100] J_{1}$, the spin-wave spectrum broadens to such an extent that there is barely any dispersion left. This featureless incoherent part of the spin-wave spectrum should experimentally be difficult to distinguish from the background. Therefore, the observed spin-wave dispersions in inelastic neutron scattering most likely corresponds to the coherent part of the spin-wave spectrum in our theoretical calculations. To further illustrate the coherent and incoherent portions of the spin-wave spectrum for the MgO-HEO model, Fig. 9 in Appendix D presents energy distribution curves for eight fixed momenta near the $q=(1 / 2,1 / 2,1 / 2)$ AFM ordering wave vector. At this point, we do not have an explanation for the origin of the $\approx 10 J_{1}$ energy scale at which the sharp dispersion and the broad continuum separate. Since a large concentration of spin vacancies cannot, to the best of our knowledge, be treated perturbatively, we do not know how to derive an analytical formula for the coherent-incoherent separation energy.

Note that for the spin-wave spectra of our disordered models (see Figs. 4-6, 9, and 10), a logarithmic scale is used for the spin-wave intensity. We also note that the sizes of the supercell Brillouin zones are strongly reduced with respect to the size of the normal cell Brillouin zone corresponding to the primitive cell of the $q=(1 / 2,1 / 2,1 / 2)$ AFM state (see Fig. 7). This leads to the formation of numerous spin-wave branches and gap openings. As the supercells become larger, these extra bands merge to form the physical broadenings of the spin-wave dispersions. This is closely related to disorder induced broadenings of electron and phonon bands simulated via supercell unfolding [43-46]. 


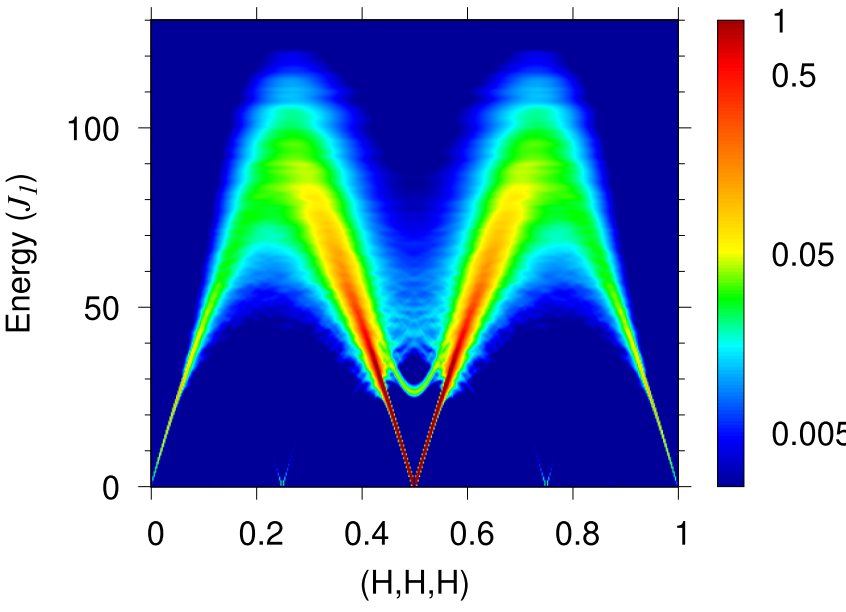

FIG. 5. Spin-wave spectrum of the $\mathrm{Co}_{0.33} \mathrm{Ni}_{0.33} \mathrm{Cu}_{0.33} \mathrm{O}$ fcc lattice spin model with moment size disorder and without spin vacancy disorder, obtained from averaging over 100 nonorthogonal supercells with $250 \mathrm{fcc}$ lattice sites on average and $J_{2}=-15 J_{1}, \Delta=0.5$, and $K=0$.

Next we dissect the consequences of the two different types of disorder in our $\mathrm{MgO}-\mathrm{HEO}$ model: the moment size disorder induced by the $\mathrm{Co}, \mathrm{Ni}$, and $\mathrm{Cu}$ cations and the spin-vacancy disorder induced by the $\mathrm{Mg}$ and $\mathrm{Zn}$ cations. In Fig. 5 we focus on the moment size disorder by presenting the spin-wave spectrum of the $\mathrm{Co}_{0.33} \mathrm{Ni}_{0.33} \mathrm{Cu}_{0.33} \mathrm{O}$ model in which there is only moment size disorder, but no spin-vacancy disorder. We note two differences between Figs. 4 and 5. First, the low energy part of the spectrum of the $\mathrm{Co}_{0.33} \mathrm{Ni}_{0.33} \mathrm{Cu}_{0.33} \mathrm{O}$ model is much sharper than for our MgO-HEO model. This illustrates that the scattering of low energy spin waves against moment size disorder is strongly suppressed as a function of energy. The situation is analogous to the case of low energy acoustic phonons scattering against mass disorder, for which in three dimensions the mass perturbation is proportional to the quadratic power of the energy; see Ref. [47] and references

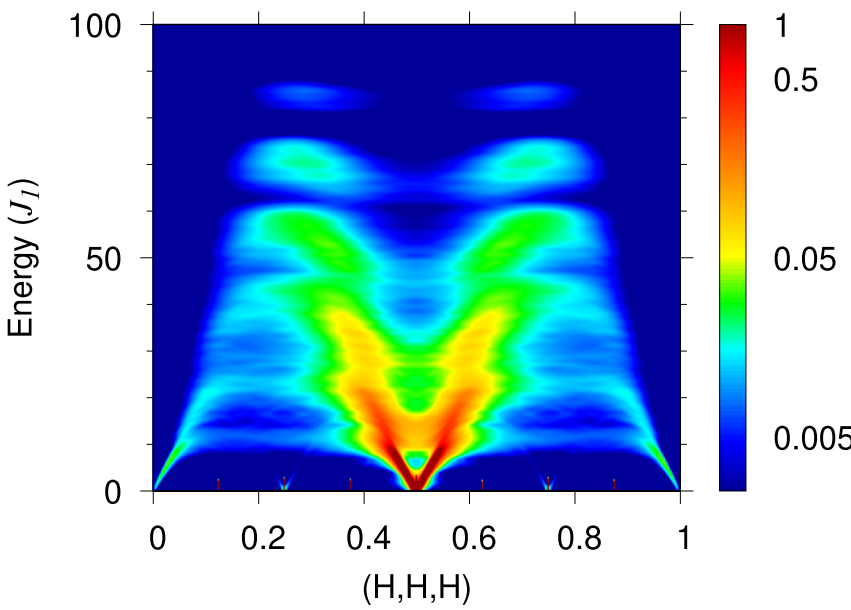

FIG. 6. Spin-wave spectrum of the $\mathrm{Ni}_{0.6} \mathrm{Mg}_{0.4} \mathrm{O}$ fcc lattice spin model without moment size disorder and with spin vacancy disorder obtained from averaging over 100 nonorthogonal supercells with 250 fcc lattice sites on average and $J_{2}=-15 J_{1}, \Delta=0.5$, and $K=0$.

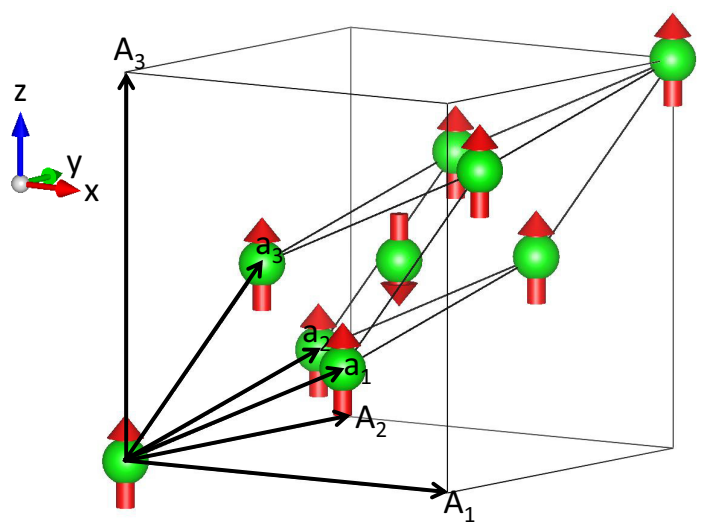

FIG. 7. Primitive cell of the $q=(1 / 2,1 / 2,1 / 2)$ AFM configuration spanned by $a_{1}, a_{2}$, and $a_{3}$ imbedded in the corresponding orthogonal conventional cell spanned by $A_{1}=3 a_{1}-a_{2}-a_{3}, A_{2}=$ $-a_{1}+3 a_{2}-a_{3}$, and $A_{3}=-a_{1}-a_{2}+3 a_{3}$

therein. A second difference is that the magnon propagation velocity, that is the slope of the low energy spin-wave band close to the $q=(1 / 2,1 / 2,1 / 2)$ AFM ordering wave vector, is significantly higher. Similarly, the maximum energy of the spin-wave intensity is also higher. The reason for the reduction of the magnon propagation velocity and the maximum energy of the spin-wave intensity in the $\mathrm{MgO}-\mathrm{HEO}$ is the presence of the spin vacancies, which make it energetically less costly for the remaining spins to rotate away from the AFM ground state.

In Fig. 6 the spin-wave excitation spectrum for the $\mathrm{Ni}_{0.6} \mathrm{Mg}_{0.4} \mathrm{O}$ model is presented. In this model there is an equal concentration of disordered spin vacancies as in the $\mathrm{MgO}-\mathrm{HEO}$ model, but no moment size disorder. The magnon propagation velocities and the maximum energy of the spinwave intensity in Figs. 4 and 6 are quite similar. Moreover, the spin-wave excitation spectrum for the $\mathrm{Ni}_{0.6} \mathrm{Mg}_{0.4} \mathrm{O}$ model is coherent roughly within $[0,10] J_{1}$ and incoherent within $[10,100] J_{1}$, just as for the MgO-HEO model. One conspicuous difference is that the incoherent part of the spin-wave excitation spectrum of $\mathrm{Ni}_{0.6} \mathrm{Mg}_{0.4} \mathrm{O}$ shows a number of strong gaps and kinks. Diluted fcc antiferromagnets have been realized experimentally. For example, $q=(1 / 2,1 / 2,1 / 2)$ AFM order in the presence of large spin-vacancy concentrations has been observed in $\mathrm{Ni}_{1-x} \mathrm{Mg}_{x} \mathrm{O}$ [23], $\mathrm{Ni}_{1-x} \mathrm{Zn}_{x} \mathrm{O}$ [24], and $\mathrm{Co}_{1-x} \mathrm{Mg}_{x} \mathrm{O}$ [25]. It would be interesting if the kinks and gaps in our $\mathrm{Ni}_{0.6} \mathrm{Mg}_{0.4} \mathrm{O}$ simulations could be observed experimentally. The nature of the kinks and gaps in the $\mathrm{Ni}_{0.6} \mathrm{Mg}_{0.4} \mathrm{O}$ originates from the different distributions of $\mathrm{Mg}$ spin vacancies around the local Ni spins. Specifically, Fig. 10 shows that the gap structure in the spin-wave excitation spectrum correlates with the number of next-nearest-neighbor $\mathrm{Mg}$ spin vacancies that surround the $\mathrm{Ni}$ spins.

Finally, we investigate the influence of magnetic disorder on the spin-wave gap. Table II lists the spin gaps induced by an easy-axis anisotropy $K=0.05 J_{1}$ for various model systems. For the $\mathrm{CoO}, \mathrm{NiO}$, and $\mathrm{CuO}$ models without disorder, we see that the spin-wave gap is proportional to the size of the cation moments, as expected. To extract the spin gap 
TABLE II. Spin gaps induced by easy-axis anisotropy $K=$ $0.05 J_{1}$ for ordered and disordered fcc lattice spin models with various cation compositions with $J_{2}=-15 J_{1}$ and $\Delta=0.5$.

\begin{tabular}{lc}
\hline \hline & Spin gap $\left(J_{1}\right)$ \\
\hline $\mathrm{CoO}, \mathrm{NiO}, \mathrm{CuO}$ & $6.26,4.17,2.09$ \\
$\mathrm{Co}_{0.2} \mathrm{Ni}_{0.2} \mathrm{Cu}_{0.2} \mathrm{Mg}_{0.2} \mathrm{Zn}_{0.2} \mathrm{O}$ & 2.47 \\
$\mathrm{Co}_{0.33} \mathrm{Ni}_{0.33} \mathrm{Cu}_{0.33} \mathrm{O}$ & 4.32 \\
$\mathrm{Ni}_{0.6} \mathrm{Mg}_{0.4} \mathrm{O}$ & 2.55 \\
\hline \hline
\end{tabular}

for our disordered models, we fit the spin-wave excitation spectrum at the $q=(1 / 2,1 / 2,1 / 2)$ AFM ordering wave vector with a Gaussian. The spin-wave gap that we obtain for $\mathrm{Co}_{0.33} \mathrm{Ni}_{0.33} \mathrm{Cu}_{0.33} \mathrm{O}$ is nearly equal to that for our $\mathrm{NiO}$ model. This suggests that the the spin-wave gap is proportional to the average moment size: $\frac{1}{3}(3 / 2+1+1 / 2)=1$. This is further confirmed by the fact that the spin-wave gaps of the $\mathrm{MgO}$ HEO and the $\mathrm{Ni}_{0.6} \mathrm{Mg}_{0.4} \mathrm{O}$ model are close in energy. For these two models we also notice that the spin gap is roughly proportional to $(1-x)$ with $x$ the vacancy concentration. For example, the spin-wave gap of $\mathrm{NiO}$ multiplied by the moment concentration is $0.6 \times 4.17=2.51$, i.e., approximately equal to the spin-wave gap of $\mathrm{Ni}_{0.6} \mathrm{Mg}_{0.4} \mathrm{O}$ of 2.57 , and of $\mathrm{Mg}$-HEO of 2.43. Overall, we find that the spin gap in our model is roughly proportional to the average moment size if we count the spin vacancies as having zero magnetic moment. Most likely, the reason for this simple behavior lies in the long wavelengths of the gapped spin waves, which average out the effects of disorder.

\section{DISCUSSION}

In our paper we have qualitatively modeled the magnetic ground state and spin-wave excitations in $\mathrm{MgO}-\mathrm{HEO}$ with a rhombohedrally distorted fcc spin model that depends on four global parameters and fully takes into account the moment size and spin-vacancy disorder. One aspect not modeled here and to be considered in future studies is the influence of disorder in the exchange couplings on the spin-wave excitations. Recently, the exchange couplings in MgO-HEO have been computed from first principles [32]. From fitting the total energy of three magnetic configurations in six ordered cation distributions, the ratio of the second- to first-nearest-neighbor exchange couplings was found to vary from $J_{2}=-11.4 J_{1}$ for $\mathrm{Ni}-\mathrm{Ni}$ pairs to $J_{2}=-2.6 J_{1}$ for $\mathrm{Cu}-\mathrm{Cu}$ pairs, with an average ratio of $J_{2}=-6.6 J_{1}$. This is reasonably consistent with our conclusion that $J_{2}=-5 J_{1}$ is needed to stabilize a significant portion of the disordered configurations in the $q=$ $(1 / 2,1 / 2,1 / 2)$ AFM ground state. In this same study [32] an average fitting of the exchange coupling was performed via calculations in a large disordered supercell and led to the conclusion that $J_{2}=-15.1 J_{1}$, very close to the value used in our paper to study spin-wave excitations. Another aspect that would be significant to consider is the influence of local distortions on the exchange couplings. Local Jahn-Teller distortions of the oxygen anions surrounding the $\mathrm{Cu}^{2+}$ cations have been observed in $\mathrm{MgO}-\mathrm{HEO}$ via extended x-ray absorption fine structure spectroscopy [26], electron paramagnetic resonance, and x-ray diffraction studies [48]. From DFT [49] it is concluded that these Jahn-Teller distortions are randomly orientated. Such distortions could induce a large spread in the exchange couplings even for first- or second-nearest-neighbor couplings of a fixed interspecies pair. For example, for the high entropy alloy $\mathrm{CrFeCoNi}$, DFT calculations show that the nearest-neighbor $\mathrm{Fe}-\mathrm{Fe}$ interactions can fluctuate roughly between +10 and $-10 \mathrm{meV}$ depending on the local chemical environment [50]. A realistic spin model can be benchmarked against inelastic neutron scattering. Features that can be used to constrain the model are the experimentally observed spinwave gap of $7 \mathrm{meV}$ [22] and the magnon propagation velocity [18]. In addition, our current paper has found that the spinwave excitations in $\mathrm{MgO}-\mathrm{HEO}$ consist of a coherent part and a featureless incoherent part. Inelastic neutron scattering experiments on $\mathrm{MgO}-\mathrm{HEO}$ are most likely only able to resolve the coherent portion of the spin-wave spectrum. The maximum of the coherent spectra (in our current simulations roughly equal to $10 J_{1}$ ) is an additional quantitative aspect of the spin-wave spectrum that can be benchmarked against inelastic neutron scattering. In order to make detailed comparisons against the experiments, powder averaging will need to be taken into account. Performing a spherical average over the momenta in combination with the treatment of strong disorder will be a computational challenge.

Another interesting aspect to consider in future investigations is the magnetic frustration induced by competition between the first- and second-nearest-neighbor exchange couplings. In this paper we have worked under the assumption that below the Néel temperature, $\mathrm{MgO}-\mathrm{HEO}$ orders in the $q=(1 / 2,1 / 2,1 / 2)$ AFM ground state. This is what has been reported based on neutron diffraction studies on $\mathrm{MgO}-\mathrm{HEO}$ and it is also the ground state that has been experimentally observed for the end members $\mathrm{NiO}$ and $\mathrm{CoO}[20,21]$. However, it is possible that no global rhombohedral distortion takes place. Indeed, as of yet, no global distortion has been observed experimentally, although this could also be due to limited resolution. In the absence of a rhombohedral distortion, the AFM ground state of $\mathrm{MgO}-\mathrm{HEO}$ could be a mixture of the four ordering wave vectors $q=(1 / 2,1 / 2,1 / 2),(-1 / 2,1 / 2,1 / 2)$, $(1 / 2,-1 / 2,1 / 2)$, and $(1 / 2,1 / 2,-1 / 2)$ as is the case for the ordered fcc model with weak nearest FM and strong next-nearest AFM couplings [35]. Note that such a ground state is not a mixture of single $q$ domains; even within the domains the four $q$ vectors are mixed. The AFM ground state has been resolved from powder diffraction for which it is not possible to distinguish between the single or the mixed $q$ state. Interestingly, in the absence of a rhombohedral distortion, the first-nearest-neighbor interaction is frustrated. As can be seen in Fig. 1, half of the nearest FM exchange couplings, labeled $J_{1}^{\prime}$, are frustrated in the $q=(1 / 2,1 / 2,1 / 2)$ configuration. A moderate frustration, expressed as the ratio of the Curie-Weiss temperature over the Neel temperature, has in fact been reported for MgO-HEO [19]. Frustration of the nearest-neighbor interaction could possibly also explain some of the other curious magnetic properties of $\mathrm{MgO}-\mathrm{HEO}$, such as for example the broad phase transition ranging from 140

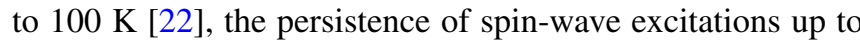
room temperature [18], and the bifurcation of the field cooled and zero field cooled magnetic susceptibility [18,19]. 


\section{CONCLUSION}

In this paper we have investigated the spin-wave excitations in the high entropy oxide $\mathrm{Mg}_{0.2} \mathrm{Co}_{0.2} \mathrm{Ni}_{0.2} \mathrm{Cu}_{0.2} \mathrm{Zn}_{0.2} \mathrm{O}$ (MgO-HEO). To model the magnetic interactions in $\mathrm{MgO}-\mathrm{HEO}$, we utilize a fcc spin model with FM nearest- and AFM next-nearest-neighbor interactions in the presence of a rhombohedral distortion and magnetic anisotropy. The Co, $\mathrm{Ni}$, and $\mathrm{Cu}$ cations are modeled as spins with a moment size of $3 / 2,1$, and $1 / 2$, respectively, whereas the $\mathrm{Mg}$ and $\mathrm{Zn}$ cations are taken to be spin vacancies. We employ linear spin-wave theory in combination with the supercell approximation to treat the disorder in order to theoretically investigate the ground state and the spin-wave excitations. Stabilizing the experimentally reported $q=(1 / 2,1 / 2,1 / 2)$ AFM ground state in our disordered model for $\mathrm{MgO}-\mathrm{HEO}$ requires significantly larger second-nearest-neighbor interactions and rhombohedral distortions compared to the case without disorder. The calculated spin-wave spectrum of $\mathrm{MgO}-\mathrm{HEO}$ consists of a coherent dispersive part at low energies, whereas at higher energies the spectrum is incoherent due to the disorder induced broadening. To differentiate the influence of moment size disorder and the spin-vacancy disorder we compute the spin-wave spectrum of $\mathrm{Co}_{0.33} \mathrm{Ni}_{0.33} \mathrm{Cu}_{0.33} \mathrm{O}$ and $\mathrm{Ni}_{0.6} \mathrm{Mg}_{0.4} \mathrm{O}$ and find these to be qualitatively different from each other and from the one of MgO-HEO. We also investigated the spin-wave gap for various cation compositions and find that for the considered model, it is proportional to the average moment size per lattice site.

\section{ACKNOWLEDGMENTS}

T.B., D.S.P., R.P.H., and R.S.F. were supported by the U.S. Department of Energy (DOE), Office of Science, Basic Energy Sciences, Materials Sciences and Engineering Division. We used resources of the National Energy Research Scientific Computing Center, a DOE Office of Science User Facility supported by the Office of Science of the U.S. DOE under Contract No. DE-AC02-05CH11231. G.A. was supported by the scientific Discovery through Advanced Computing program funded by U.S. Department of Energy, Office of Science, Advanced Scientific Computing Research and Basic Energy Sciences, Division of Materials Sciences and Engineering. Part of this research was conducted at the Center for Nanophase Materials Sciences, which is a DOE Office of Science User Facility. This manuscript has been authored by UT-Battelle, LLC under Contract No. DE-AC05-00OR22725 with the U.S. Department of Energy. The United States Government retains and the publisher, by accepting the article for publication, acknowledges that the United States Government retains a non-exclusive, paid-up, irrevocable, world-wide license to publish or reproduce the published form of this manuscript, or allow others to do so, for United States Government purposes. The Department of Energy will provide public access to these results of federally sponsored research in accordance with the DOE Public Access Plan [51].

\section{APPENDIX A: DIFFERENTIATING SPIN CONFIGURATIONS}

To quantify how close the ground state of a disordered supercell is to the $q=(1 / 2,1 / 2,1 / 2)$ AFM configuration, one could compute the $q=(1 / 2,1 / 2,1 / 2)$ AFM energy relative to the ground state energy. However, for comparing among different parameter sets, the energy is not a good measure because for a given configuration the energy varies as a function of the parameters. Therefore, instead we use the spin configuration of the ground state to determine its closeness to the $q=(1 / 2,1 / 2,1 / 2)$ AFM configuration. To this end, we define the difference between two spin configurations as the absolute difference of the spin vectors averaged over the spins in the configurations. While doing this, we have to be mindful of the rotational invariance when computing this difference. For example, in the isotropic case the difference between two spin configurations should not depend on an overall rotation of either of the spin configurations. To this end, we define the difference between spin configurations $A$ and $B$ as follows. We first perform an overall rotation of configuration $B$ such that its first spin aligns as much as possible with the first spin of configuration $A$ and then compute the average absolute difference of the spin vectors. To not bias our measure of difference toward the first spin, we also repeat the procedure with aligning each of the other spins as much as possible. Following the above, the difference of spin configurations $B$ and $A$ is given by

$$
\Delta S^{A, B}=\frac{1}{N^{2}} \sum_{r, r^{\prime}}\left|S_{r}^{A}-S_{r, r^{\prime}}^{A, B}\right|
$$

where $N$ is the total number of spins, $S_{r}^{A}$ is the spin vector of spin $r$ in configuration $A$, and

$$
S_{r, r^{\prime}}^{A, B}=U_{r^{\prime}}^{A, B} S_{r}^{B}
$$

is the spin vector of spin $r$ in configuration $B$ that is rotated to align its spin $r^{\prime}$ as much as possible with the corresponding spin $r^{\prime}$ in configuration $A$. For isotropic interactions, the rotation matrix is an element of the three-dimensional rotation group: $U_{r^{\prime}}^{A} \in \mathrm{SO}(3)$. In that case, the spin $r^{\prime}$ of configuration $A$ and $B$ can be perfectly aligned. In the presence of an easy-axis anisotropy $K>0$, the rotation matrix is an element of the product of the group of 2D rotations around the easy axis and the group of reflections in the plane perpendicular to the easy axis : $U_{r^{\prime}}^{A} \in \mathrm{SO}(2) \times Z_{2}$. For example, suppose we have an easy axis along the $z$ direction and we have two spins: $S_{A}=\left(\cos \left(\phi_{A}\right) \sin \left(\theta_{A}\right), \sin \left(\phi_{A}\right) \sin \left(\theta_{A}\right), \cos \left(\theta_{A}\right)\right)$ and $\quad S_{B}=\left(\cos \left(\phi_{B}\right) \sin \left(\theta_{B}\right), \sin \left(\phi_{B}\right) \sin \left(\theta_{B}\right), \cos \left(\theta_{B}\right)\right)$. In that case the rotation matrix $U^{A, B}$ rotates $S_{B}$ to $\left(\cos \left(\phi_{A}\right) \sin \left(\theta_{B}^{\prime}\right), \sin \left(\phi_{A}\right) \sin \left(\theta_{B}^{\prime}\right), \cos \left(\theta_{B}^{\prime}\right)\right) \quad$ with $\theta_{B}^{\prime} \quad$ such that $\operatorname{sgn}\left(\cos \left(\theta_{B}^{\prime}\right)\right)=\operatorname{sgn}\left(\cos \left(\theta_{A}\right)\right)$ and $\left|\cos \left(\theta_{B}^{\prime}\right)\right|=\left|\cos \left(\theta_{B}\right)\right|$.

Having defined a measure to differentiate two spin configurations, we have to choose a cutoff below which we consider two spin configurations to be equal. In this paper, we consider a spin configuration $A$ to have the $q=(1 / 2,1 / 2,1 / 2)$ AFM ground state if $\Delta S<10^{-4}$. For example, the configuration in Fig. 2(b) [Fig. 2(a)] has $\Delta S$ equal to 0.00002 (2.126 36) and therefore is considered to be (not to be) in the $q=(1 / 2,1 / 2,1 / 2)$ AFM ground state.

\section{APPENDIX B: NONORTHOGONAL SUPERCELLS}

To better average the disorder configurations we not only randomly distribute the cations within the supercells, but also randomly change the shapes of the supercells themselves [37]. 


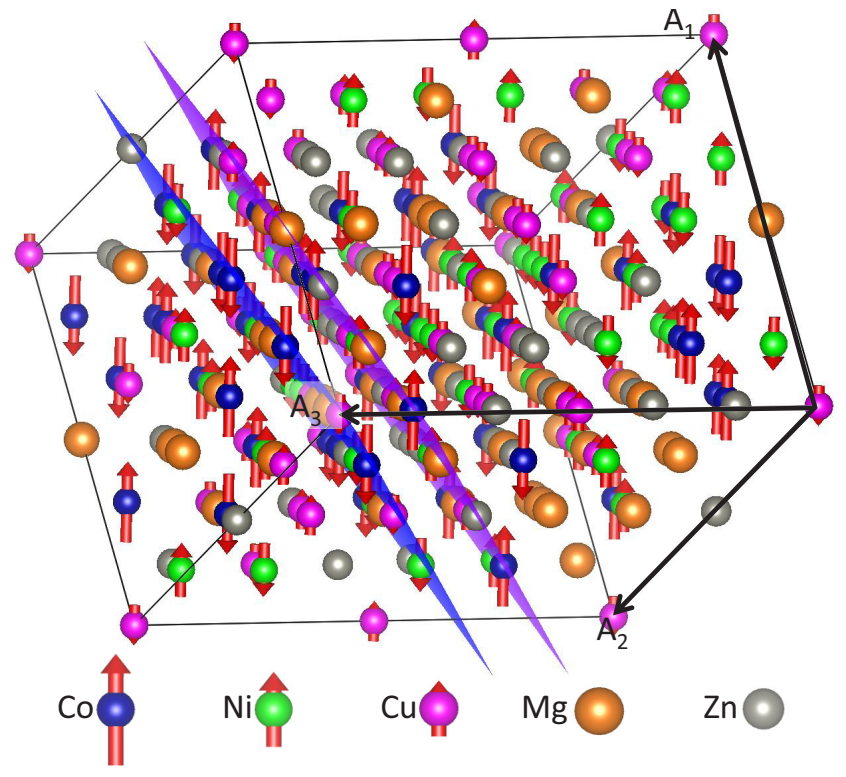

FIG. 8. One of the nonorthogonal supercells used for computing the disorder-configuration averaged spin-wave spectra.

To construct the nonorthogonal supercells, we first consider the primitive cell of the antiferromagnetic order of the second kind with ordering wave vector $q=(1 / 2,1 / 2,1 / 2)$ illustrated in Fig. 7.

We construct nonorthogonal supercells that are supercells relative to the primitive cell of the $q=(1 / 2,1 / 2,1 / 2)$ AFM configuration. We note that while these resulting nonorthogonal supercells satisfy the boundary conditions of the $q=(1 / 2,1 / 2,1 / 2)$ AFM configuration, they do not satisfy the boundary conditions of the $q=(-1 / 2,1 / 2,1 / 2)$, $(1 / 2,-1 / 2,1 / 2)$, and $(1 / 2,1 / 2,-1 / 2)$ supercells. As a result, the $q=(1 / 2,1 / 2,1 / 2)$ AFM configuration is significantly more stable in these nonorthogonal supercells compared to the orthogonal ones used to determine the ground state configuration in Sec. III. We select the sizes of these supercells to be within 115 and $135 q=(1 / 2,1 / 2,1 / 2)$ AFM primitive cells, corresponding to 230 and 270 cation sites. We allow the unit cell angles to vary between 80 and $100^{\circ}$. In Fig. 8 we illustrate one of the nonorthogonal supercells used for computing the disorder-configuration averaged spin- wave spectra. It is spanned by $A_{1}=-a_{1}+5 a_{2}-5 a_{3}, A_{2}=$ $-4 a_{1}+4 a_{2}+2 a_{3}$, and $A_{3}=5 a_{1}+a_{2}-3 a_{3}$. It contains 248 cations and its unit cell angles are $\alpha=83.1354, \beta=90$, and $\gamma=85.904$.

\section{APPENDIX C: ADDITIONAL GROUND STATE RESULTS}

Tables III and IV show that the number of configurations, for which the $q=(1 / 2,1 / 2,1 / 2)$ AFM ground state is realized, is essentially the same for the isotropic case $(K=0)$ and the anisotropic case $\left(K=0.05 J_{1}\right)$, respectively.

TABLE III. Probability of an orthogonal $\mathrm{Co}_{6} \mathrm{Ni}_{6} \mathrm{Cu}_{6} \mathrm{Mg}_{6} \mathrm{Zn}_{8}$ supercell being in the $q=(1 / 2,1 / 2,1 / 2)$ AFM ground state in the case of isotropic interactions: $K=0$, obtained from simulating 100 $\mathrm{Co}_{6} \mathrm{Ni}_{6} \mathrm{Cu}_{6} \mathrm{Mg}_{6} \mathrm{Zn}_{8}$ supercells.

\begin{tabular}{ccccc}
\hline \hline & $\begin{array}{c}J_{2}=-J_{1} \\
(\%)\end{array}$ & $\begin{array}{c}J_{2}=-2 J_{1} \\
(\%)\end{array}$ & $\begin{array}{c}J_{2}=-5 J_{1} \\
(\%)\end{array}$ & $\begin{array}{c}J_{2}=-15 J_{1} \\
(\%)\end{array}$ \\
\hline$\Delta=0.0$ & 0 & 0 & 0 & 1 \\
$\Delta=0.1$ & 0 & 0 & 6 & 9 \\
$\Delta=0.2$ & 0 & 4 & 20 & 23 \\
$\Delta=0.5$ & 27 & 47 & 63 & 68 \\
\hline \hline
\end{tabular}

TABLE IV. Probability of an orthogonal $\mathrm{Co}_{6} \mathrm{Ni}_{6} \mathrm{Cu}_{6} \mathrm{Mg}_{6} \mathrm{Zn}_{8}$ supercell being in the $q=(1 / 2,1 / 2,1 / 2)$ AFM ground state in the case of anisotropic interactions: $K=0.05 J_{1}$, obtained from simulating $100 \mathrm{Co}_{6} \mathrm{Ni}_{6} \mathrm{Cu}_{6} \mathrm{Mg}_{6} \mathrm{Zn}_{8}$ supercells.

\begin{tabular}{ccccc}
\hline \hline & $\begin{array}{c}J_{2}=-J_{1} \\
(\%)\end{array}$ & $\begin{array}{c}J_{2}=-2 J_{1} \\
(\%)\end{array}$ & $\begin{array}{c}J_{2}=-5 J_{1} \\
(\%)\end{array}$ & $\begin{array}{c}J_{2}=-15 J_{1} \\
(\%)\end{array}$ \\
\hline$\Delta=0.0$ & 0 & 0 & 1 & 2 \\
$\Delta=0.1$ & 0 & 1 & 6 & 14 \\
$\Delta=0.2$ & 1 & 10 & 18 & 22 \\
$\Delta=0.5$ & 33 & 49 & 64 & 70 \\
\hline \hline
\end{tabular}




\section{APPENDIX D: ADDITIONAL SPIN-WAVE EXCITATION RESULT}

Figures 9 shows eight energy distribution curves for fixed momenta near the $q=(1 / 2,1 / 2,1 / 2)$ AFM ordering wave vector, to further illustrate the coherent and incoherent portions of the spin-wave spectrum for the MgO-HEO model. Fig. 10 shows that the gap structure in the spin-wave excitation spectrum correlates with the number of next-nearest neighbor $\mathrm{Mg}$ spin vacancies that surround the $\mathrm{Ni}$-spins.

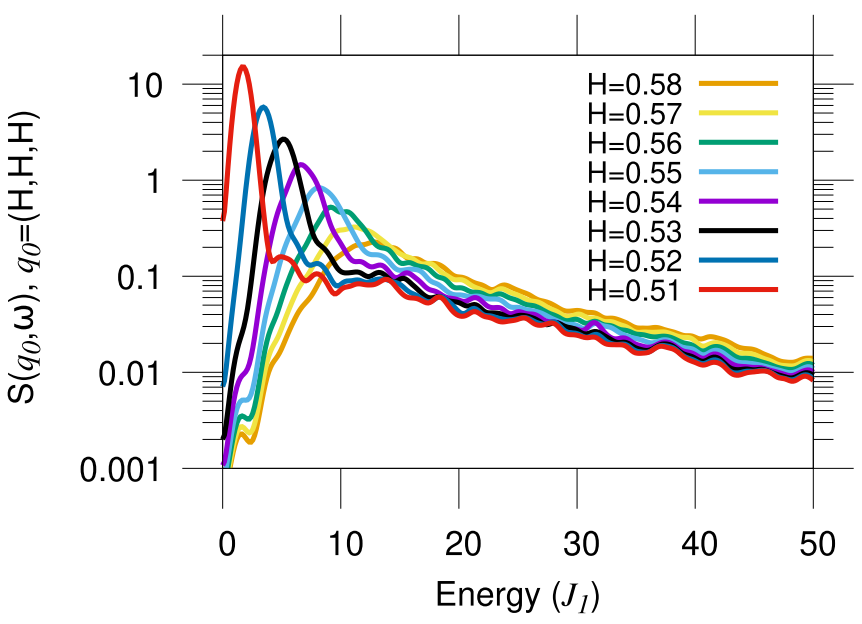

FIG. 9. Energy distribution curves of the spin-wave spectrum of the disordered $\mathrm{Mg}_{0.2} \mathrm{Co}_{0.2} \mathrm{Ni}_{0.2} \mathrm{Cu}_{0.2} \mathrm{Zn}_{0.2} \mathrm{O}$ fcc lattice spin model obtained from averaging over 100 nonorthogonal supercells with 250 fcc lattice sites on average and $J_{2}=-15 J_{1}, \Delta=0.5$, and $K=0$.
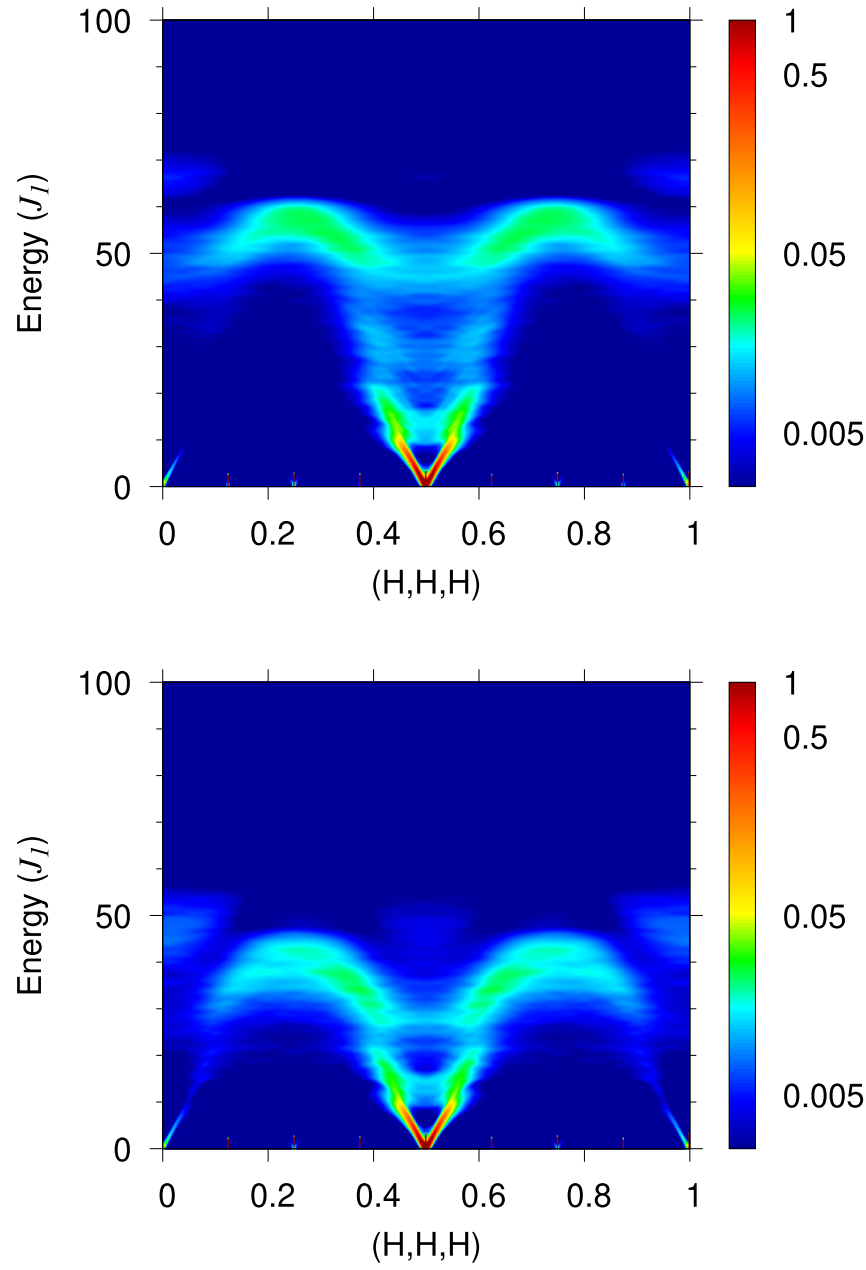

FIG. 10. Spin-wave spectrum of the $\mathrm{Ni}_{0.6} \mathrm{Mg}_{0.4} \mathrm{O}$ fcc lattice spin model without moment size disorder and with spin vacancy disorder obtained from averaging over 100 nonorthogonal supercells with 250 fcc lattice sites on average and $J_{2}=-15 J_{1}, \Delta=0.5$, and $K=0$, projected on spins that have two (upper panel) and three (lower panel) next-nearest-neighbor spin vacancies.
[1] D. B. Miracle and O. N. Senkov, A critical review of high entropy alloys and related concepts, Acta Mater. 122, 448 (2017).

[2] C. M. Rost, E. Sachet, T. Borman, A. Moballegh, E. C. Dickey, D. Hou, J. L. Jones, S. Curtarolo, and J.-P. Maria, Entropystabilized oxides, Nat. Commun. 6, 8485 (2015).

[3] A. Sarkar, Q. Wang, A. Schiele, M. R. Chellali, S. S. Bhattacharya, D. Wang, T. Brezesinski, H. Hahn, L. Velasco, and B. Breitung, High-entropy oxides: Fundamental aspects and electrochemical properties, Adv. Mater. 31, 1806236 (2019).

[4] D. Bérardan, S. Franger, D. Dragoe, A. K. Meena, and N. Dragoe, Colossal dielectric constant in high entropy oxides, Phys. Status Solidi RRL 10, 328 (2016).

[5] D. Bérardan, S. Franger, A. K. Meena, and N. Dragoe, Room temperature lithium superionic conductivity in high entropy oxides, J. Mater. Chem. A 4, 9536 (2016).
[6] J. L. Braun, C. M. Rost, M. Lim, A. Giri, D. H. Olson, G. N. Kotsonis, G. Stan, D. W. Brenner, J.-P. Maria, and P. E. Hopkins, Charge-induced disorder controls the thermal conductivity of entropy-stabilized oxides, Adv. Mater. 30, 1805004 (2018).

[7] A. Sarkar, R. Djenadic, D. Wang, C. Hein, R. Kautenburger, O. Clemens, and H. Hahn, Rare earth and transition metal based entropy stabilised perovskite type oxides, J. Eur. Ceram. Soc. 38, 2318 (2018).

[8] S. Jiang, T. Hu, J. Gild, N. Zhou, J. Nie, M. Qin, T. Harrington, K. Vecchio, and J. Luo, A new class of high-entropy perovskite oxides, Scr. Mater. 142, 116 (2018).

[9] Y. Sharma, B. L. Musico, X. Gao, C. Hua, A. F. May, A. Herklotz, A. Rastogi, D. Mandrus, J. Yan, H. N. Lee, M. F. Chisholm, V. Keppens, and T. Z. Ward, Single-crystal high 
entropy perovskite oxide epitaxial films, Phys. Rev. Mater. 2, 060404(R) (2018).

[10] J. Gild, M. Samiee, J. L. Braun, T. Harrington, H. Vega, P. E. Hopkins, K. Vecchio, and J. Luo, High-entropy fluorite oxides, J. Eur. Ceram. Soc. 38, 3578 (2018).

[11] K. Chen, X. Pei, L. Tang, H. Cheng, Z. Li, C. Li, X. Zhang, and L. An, A five-component entropy-stabilized fluorite oxide, J. Eur. Ceram. Soc. 38, 4161 (2018).

[12] J. Dąbrowa, M. Stygar, A. Mikuła, A. Knapik, K. Mroczka, W. Tejchman, M. Danielewski, and M. Martin, Synthesis and microstructure of the $(\mathrm{Co}, \mathrm{Cr}, \mathrm{Fe}, \mathrm{Mn}, \mathrm{Ni})_{3} \mathrm{O}_{4}$ high entropy oxide characterized by spinel structure, Mater. Lett. 216, 32 (2018).

[13] E. Castle, T. Csanádi, S. Grasso, J. Ã. Dusza, and M. Reece, Processing and properties of high-entropy ultra-high temperature carbides, Sci. Rep. 8, 8609 (2018).

[14] X. Yan, L. Constantin, Y. Lu, J.-F. Silvain, M. Nastasi, and B. Cui, $\left(\mathrm{Hf}_{0.2} \mathrm{Zr}_{0.2} \mathrm{Ta}_{0.2} \mathrm{Nb}_{0.2} \mathrm{Ti}_{0.2}\right) \mathrm{C}$ high-entropy ceramics with low thermal conductivity, J. Am. Ceram. Soc. 101, 4486 (2018).

[15] J. Gild, Y. Zhang, T. Harrington, S. Jiang, T. Hu, M. C. Quinn, W. M. Mellor, N. Zhou, K. Vecchio, and J. Luo, High-entropy metal diborides: A new class of high-entropy materials and a new type of ultrahigh temperature ceramics, Sci. Rep. 6, 37946 (2016).

[16] Z. Deng, A. Olvera, J. Casamento, J. S. Lopez, L. Williams, R. Lu, G. Shi, P. F. P. Poudeu, and E. Kioupakis, Semiconducting high-entropy chalcogenide alloys with ambi-ionic entropy stabilization and ambipolar doping, Chem. Mater. 32, 6070 (2020).

[17] P. B. Meisenheimer, T. J. Kratofil, and J. T. Heron, Giant enhancement of exchange coupling in entropy-stabilized oxide heterostructures, Sci. Rep. 7, 13344 (2017).

[18] J. Zhang, J. Yan, S. Calder, Q. Zheng, M. A. McGuire, D. L. Abernathy, Y. Ren, S. H. Lapidus, K. Page, H. Zheng, J. W. Freeland, J. D. Budai, and R. P. Hermann, Long-range antiferromagnetic order in a rocksalt high entropy oxide, Chem. Mater. 31, 3705 (2019).

[19] M. P. Jimenez-Segura, T. Takayama, D. Bérardan, A. Hoser, M. Reehuis, H. Takagi, and N. Dragoe, Long-range magnetic ordering in rocksalt-type high-entropy oxides, Appl. Phys. Lett. 114, 122401 (2019).

[20] C. G. Shull, W. A. Strauser, and E. O. Wollan, Neutron diffraction by paramagnetic and antiferromagnetic substances, Phys. Rev. 83, 333 (1951).

[21] W. L. Roth, Magnetic structures of $\mathrm{MnO}, \mathrm{FeO}, \mathrm{CoO}$, and $\mathrm{NiO}$, Phys. Rev. 110, 1333 (1958).

[22] B. A. Frandsen, K. A. Petersen, N. A. Ducharme, A. G. Shaw, E. J. Gibson, B. Winn, J. Yan, J. Zhang, M. E. Manley, and R. P. Hermann, Spin dynamics and a nearly continuous magnetic phase transition in an entropy-stabilized oxide antiferromagnet, Phys. Rev. Mater. 4, 074405 (2020).

[23] A. Z. Menshikov, Yu. A. Dorofeev, A. G. Klimenko, and N. A. Mironova, Magnetic phase diagram of $\left(\mathrm{Ni}_{1-x} \mathrm{Mg}_{x}\right) \mathrm{O}$ solid solutions, Phys. Status Solidi B 164, 275 (1991).

[24] D. Rodic, V. Spasojevic, V. Kusigerski, R. Tellgren, and H. Rundlof, Magnetic ordering in polycrystalline $\mathrm{Ni}_{x} \mathrm{Zn}_{1-x} \mathrm{O}$ solid solutions, Phys. Status Solidi B 218, 527 (2000).

[25] M. S. Seehra, J. C. Dean, and R. Kannan, Magnetic phase diagrams of diluted fcc antiferromagnets $\mathrm{Co}_{p} \mathrm{Mg}_{1-p}$ and $\mathrm{Eu}_{p} \mathrm{Sr}_{1-p} \mathrm{Te}$, Phys. Rev. B 37, 5864 (1988).
[26] C. M. Rost, Z. Rak, D. W. Brenner, and J.-P. Maria, Local structure of the $\mathrm{Mg}_{x} \mathrm{Ni}_{x} \mathrm{Co}_{x} \mathrm{Cu}_{x} \mathrm{Zn}_{x} \mathrm{O}(x=0.2)$ entropy-stabilized oxide: An exafs study, J. Am. Ceram. Soc. 100, 2732 (2017).

[27] Zs. Rak, C. M. Rost, M. Lim, P. Sarker, C. Toher, S. Curtarolo, J.-P. Maria, and D. W. Brenner, Charge compensation and electrostatic transferability in three entropy-stabilized oxides: Results from density functional theory calculations, J. Appl. Phys. 120, 095105 (2016).

[28] J. B. Goodenough, An interpretation of the magnetic properties of the perovskite-type mixed crystals $\mathrm{La}_{1-x} \mathrm{Sr}_{x} \mathrm{CoO}_{3-\lambda}$, J. Phys. Chem. Solids 6, 287 (1958).

[29] J. Kanamori, Superexchange interaction and symmetry properties of electron orbitals, J. Phys. Chem. Solids 10, 87 (1959).

[30] H.-X. Deng, J. Li, S.-S. Li, J.-B. Xia, A. Walsh, and S.-H. Wei, Origin of antiferromagnetism in coo: A density functional theory study, Appl. Phys. Lett. 96, 162508 (2010).

[31] D. Ködderitzsch, W. Hergert, W. M. Temmerman, Z. Szotek, A. Ernst, and $\mathrm{H}$. Winter, Exchange interactions in $\mathrm{NiO}$ and at the NiO(100) surface, Phys. Rev. B 66, 064434 (2002).

[32] Zs. Rák and D. W. Brenner, Exchange interactions and long-range magnetic order in the ( $\mathrm{Mg}, \mathrm{Co}, \mathrm{Cu}, \mathrm{Ni}, \mathrm{Zn}) \mathrm{O}$ entropystabilized oxide: A theoretical investigation, J. Appl. Phys. 127, 185108 (2020).

[33] M. T. Hutchings and E. J. Samuelsen, Measurement of spinwave dispersion in $\mathrm{NiO}$ by inelastic neutron scattering and its relation to magnetic properties, Phys. Rev. B 6, 3447 (1972).

[34] K. Tomiyasu and S. Itoh, Magnetic excitations in coo, J. Phys. Soc. Jpn. 75, 084708 (2006).

[35] Y. Yamamoto and T. Nagamiya, Spin arrangements in magnetic compounds of the rocksalt crystal structure, J. Phys. Soc. Jpn. 32, 1248 (1972).

[36] R. Fishman, J. Fernandez-Baca, and T. Room, Spin-Wave Theory and its Applications to Neutron Scattering and $\mathrm{THz}$ Spectroscopy (Morgan \& Claypool, San Rafael, 2018).

[37] T. Berlijn, Effects of Disordered Dopants on the Electronic Structure of Functional Materials: Wannier Function-Based First Principles Methods for Disordered Systems, Ph.D. thesis, Stony Brook University, 2011.

[38] https://g1257.github.io/spinGlassSW.html

[39] P. Buczek, L. M. Sandratskii, N. Buczek, S. Thomas, G. Vignale, and A. Ernst, Magnons in disordered nonstoichiometric low-dimensional magnets, Phys. Rev. B 94, 054407 (2016).

[40] X. Bai, J. A. M. Paddison, E. Kapit, S. M. Koohpayeh, J.J. Wen, S. E. Dutton, A. T. Savici, A. I. Kolesnikov, G. E. Granroth, C. L. Broholm, J. T. Chalker, and M. Mourigal, Magnetic Excitations Of The Classical Spin Liquid $\mathrm{MgCr}_{2} \mathrm{O}_{4}$, Phys. Rev. Lett. 122, 097201 (2019).

[41] W. J. L. Buyers, D. E. Pepper, and R. J. Elliott, Spin waves in disordered systems. II. The dilute antiferromagnet $(\mathrm{Mn}, \mathrm{Zn}) \mathrm{F}_{2}$, J. Phys. C 6, 1933 (1973).

[42] A. M. Samarakoon, A. Banerjee, S.-S. Zhang, Y. Kamiya, S. E. Nagler, D. A. Tennant, S.-H. Lee, and C. D. Batista, Comprehensive study of the dynamics of a classical kitaev spin liquid, Phys. Rev. B 96, 134408 (2017).

[43] S. Baroni, S. de Gironcoli, and P. Giannozzi, Phonon Dispersions In $\mathrm{Ga}_{x} \mathrm{Al}_{1-x}$ As Alloys, Phys. Rev. Lett. 65, 84 (1990).

[44] T. B. Boykin and G. Klimeck, Practical application of zonefolding concepts in tight-binding calculations, Phys. Rev. B 71, 115215 (2005). 
[45] V. Popescu and A. Zunger, Effective Band Structure of Random Alloys, Phys. Rev. Lett. 104, 236403 (2010).

[46] T. Berlijn, D. Volja, and W. Ku, Can Disorder Alone Destroy the $e_{g}^{\prime}$ Hole Pockets of $\mathrm{Na}_{x} \mathrm{CoO}_{2}$ ? A Wannier Function Based First-Principles Method for Disordered Systems, Phys. Rev. Lett. 106, 077005 (2011).

[47] S. Thébaud, C. A. Polanco, L. Lindsay, and T. Berlijn, Success and breakdown of the t-matrix approximation for phonondisorder scattering, Phys. Rev. B 102, 094206 (2020).

[48] D. Berardan, A. K. Meena, S. Franger, C. Herrero, and N. Dragoe, Controlled Jahn-Teller distortion in ( $\mathrm{MgCoNiCuZn}) \mathrm{O}-$ based high entropy oxides, J. Alloys Compd. 704, 693 (2017).

[49] Zs. Rák, J.-P. Maria, and D.W. Brenner, Evidence for Jahn-Teller compression in the $(\mathrm{Mg}, \mathrm{Co}, \mathrm{Ni}, \mathrm{Cu}, \mathrm{Zn}) \mathrm{O}$ entropy-stabilized oxide: A DFT study, Mater. Lett. 217, 300 (2018).

[50] T. Fukushima, H. Katayama-Yoshida, K. Sato, M. Ogura, R. Zeller, and P. H. Dederichs, Local energies and energy fluctuations applied to the high entropy alloy crfeconi, J. Phys. Soc. Jpn. 86, 114704 (2017).

[51] http://energy.gov/downloads/doe-public-access-plan. 